\title{
Review
}

\section{Pharmacological Targeting of IRE1 in Cancer}

\author{
Diana Pelizzari Raymundo, ${ }^{1,2}$ Dimitrios Doultsinos, ${ }^{1,2,5}$ Xavier Guillory, ${ }^{3,5}$ Antonio Carlesso, ${ }^{4}$ \\ Leif A. Eriksson, ${ }^{4, *}$ and Eric Chevet (10, ${ }^{1,2, *}$
}

IRE1 $\alpha$ (inositol requiring enzyme 1 alpha) is one of the main transducers of the unfolded protein response (UPR). IRE1 a plays instrumental protumoral roles in several cancers, and high IRE1 $\alpha$ activity has been associated with poorer prognoses. In this context, IRE1 $\alpha$ has been identified as a potentially relevant therapeutic target. Pharmacological inhibition of IRE1 $\alpha$ activity can be achieved by targeting either the kinase domain or the RNase domain. Herein, the recent advances in IRE1 a pharmacological targeting is summarized. We describe the identification and optimization of IRE1 $\alpha$ inhibitors as well as their mode of action and limitations as anticancer drugs. The potential pitfalls and challenges that could be faced in the clinic, and the opportunities that IRE1 $\alpha$ modulating strategies may present are discussed.

\section{Introduction}

Cancer is the second leading cause of death globally with an estimated 18.1 million cases, and 9.6 million deaths in 2018. Based on projected population aging and growth, the global burden of cancer is set to increase by more than 60\% by 2040, reaching a predicted 29.4 million cases (World Health Organization). Chemotherapies have shown their limits in many instances, while precision oncology has shown some promise. Therefore, it is becoming apparent that the identification of new targets with therapeutic relevance, and the design of new candidate drugs to impinge on these new targets' functions is required. Accumulating evidence points toward an emerging role for endoplasmic reticulum (ER) stress (see Glossary), the unfolded protein response (UPR) and proteostasis control in cancer progression [1]. The accumulation of improperly folded proteins in the ER causes ER stress, which triggers the UPR as an adaptive mechanism to restore ER proteostasis, and maintain the functionality of the secretory pathway. The activation of the UPR leads to mRNA translation attenuation, and to upregulation of genes involved in protein folding and quality control, antioxidant responses, and lipid synthesis. However, if proteostasis restoration fails, proapoptotic programs are triggered to eliminate terminally damaged cells [2]. In the context of cancer, ER stress plays protumoral roles by allowing transformed cells to cope with intrinsic stresses such as oncogene expression [3] or aneuploidy [4,5], and with extrinsic stresses such as nutrient and oxygen deprivation. Moreover, tumor cells can be subjected to extrinsic stresses caused by anticancer treatments (irradiation and chemotherapies), and ER stress signaling confers treatment resistance to cancer cells [6-10]. This evidence points towards the UPR as an appealing target in unique or adjuvant anticancer therapeutic approaches.

\section{IRE1 a Signaling}

IRE1a, (inositol requiring enzyme 1 alpha, referred to as IRE1 hereafter,) is the most evolutionary conserved ER stress sensor. IRE1 is encoded by the ERN1 gene and it is ubiquitously expressed in mammals. It is a type I, transmembrane, ER resident protein, which bears two enzymatic activities: a serine/threonine kinase, and an endoribonuclease (RNase), in its cytosolic domain. Despite being considered as a conventional kinase protein, the only substrate known so far is IRE1 itself. When improperly folded proteins accumulate in the ER, IRE1 oligomerizes and trans-auto phosphorylates, thereby activating its RNase activity. Thus far, the most prominent

\section{Highlights}

IRE1a (inositol requiring enzyme 1 alpha), a type I endoplasmic reticulum (ER)resident transmembrane protein, exhibits both RNase and kinase activities

IRE1a is involved in several diseases such as cancer, immune, metabolic, and neurodegenerative disorders, and has thus become a relevant therapeutic target.

Pharmacological modulation of IRE1a activity, and the subsequent applications, mechanisms of action, and limitations, are key to a better understanding of what would be the best anticancer approach to use in clinical settings.

The potential pitfalls/challenges and opportunities that IRE1a modulating strategies may represent are discussed.

${ }^{1}$ Proteostasis and Cancer Team, INSERM U1242, COSS Laboratory, Université de Rennes, Rennes, France ${ }^{2}$ Centre de Lutte contre le Cancer Eugène Marquis, Rennes, France ${ }^{3}$ Institut des Science Chimiques de Rennes, CNRS UMR6226, Université de Rennes, Rennes, France ${ }^{4}$ Department of Chemistry and Molecular Biology, University of Gothenburg, Göteborg, Sweden ${ }^{5}$ These authors contributed equally to this work

${ }^{*}$ Correspondence: leif.eriksson@chem.gu.se (L.A. Eriksson) and eric.chevet@inserm.fr (E. Chevet). 
signaling output mediated by IRE1 has been identified as dependent on IRE1 RNase activity. Hence, two signaling responses are catalyzed (Figure 1): (i) The nonconventional splicing of the mRNA encoding X-box binding protein-1 (XBP1) allows the expression of XBP1 spliced (XBP1s), a potent transcription factor. This event occurs through the combined action of IRE1 RNase removal of a 26 nucleotide intron and of the tRNA ligase RtcB, which ligates the 5'-OH and 2',3'-cyclic phosphate of the remaining fragments; and (ii) the RNase-mediated cleavage of ER-bound RNA (including mRNA, miRNA, and $r R N A$ ) occurs in a process called regulated IRE1-dependent decay (RIDD) of RNA. Here the degradation is executed by cellular exonucleases following IRE1-mediated cleavage.

Beyond the signaling pathways initiated downstream of IRE1 RNase, it has been demonstrated that IRE1 also executes its functions through scaffolding roles $[11,12]$. Under irremediable ER stress, IRE1 assembles into a scaffold platform for the activation of both ASK1-JNK and NF-KB pathways. Its hyperactivation causes a decrease in RNase activity and an increase in RIDD, which induces proinflammatory and apoptotic factors, reaching a terminal UPR [13] (Figure 1A). As such, IRE1 operates as a multitasking protein that transduces signals through both catalytic and noncatalytic mechanisms.

IRE1 activity is important in tumorigenesis and aggressiveness in most cancers, such as leukemia, glioblastoma, myeloma, renal, and breast cancers, and has been associated with poor prognosis $[3,10,14,15]$. Several types of cancers displaying high levels of IRE1/XBP1 activity have a worse prognosis than those with low activity. XBP1 also promotes tumor invasion and drug resistance in cancer [8]. XBP1s is overexpressed in several types of cancer, and its overexpression is directly linked to tumorigenesis, suggesting proto-oncogenic activity [16]. In addition, IRE1-XBP1s was found to regulate $\mathbf{c}-\mathbf{M y c}$ expression, contributing to oncogenicity in prostate cancer (PCa) $[3,10]$. By contrast to the IRE1/XBP1 axis that exhibits protumorigenic signaling features, RIDD may antagonize tumor invasion and angiogenesis with no significant effect on immune cell infiltration in glioblastoma [15]. Targets of RIDD include miRNAs, ribosomal RNAs, mRNA, and ER localized mRNAs encoding membrane and secretory proteins, the latter thought to be degraded to reduce ER load upon stress. However, how IRE1 regulation evolves during the pathologic progression of disease is still a matter of debate. A plethora of outstanding questions remain to be carefully addressed before fundamental discoveries can be translated into effective IRE1-targeting therapeutics to combat diseases in the clinic (see Outstanding Questions).

\section{Small Molecules Targeting IRE1}

Pharmacological modulation of IRE1 has been the focus of several drug development strategies, and compounds targeting either the kinase domain or the RNase domain have been identified. Downstream IRE1 signaling components are also of interest to drug discovery programs. Based on structural and biochemical analyses, several kinase domains display similarities to the IRE1 kinase [17]. The RNase domain is homologous only to that of the known antiviral RNaseL, revealing a convergent evolution of their catalytic mechanism [18]. IRE1 and RNaseL, in addition to biochemical similarities in their protein kinase domain, and structural similarities in their RNase domain, share the functional consequences of their activation in initiating cellular apoptosis through JNK signaling [19]. Further advances in research narrowed the differences between the pathways. A recent report suggested a consensus cleavage site in RNaseL targets and in those mRNAs that are cleaved by IRE1 as part of the RIDD pathway [20]. The characteristics and structures of known IRE1 inhibitors are presented in Table 1, as well as in Figures 2 and 3.

Interest in targeting IRE1 as a therapeutic strategy has prompted the search for highly selective IRE1 modulators. The characterization of the allosteric relationship between the kinase and RNase domains has allowed for the modulation of IRE1 with small molecules targeting the ATP-binding site
Glossary

c-Myc: the Myc is a family of regulator genes and proto-oncogenes that code for transcription factors. The Myc family consists of three related human genes: c-Myc, I-Myc, and n-Myc. c-Myc was the first gene to be discovered in this family, due to homology with the viral gene $v$-myc.

ER stress: a condition when an increased load of unfolded proteins in the ER lumen causes a disturbance in the ER leading to an inability to restore its functions and induces cell death, which is usually in the form of apoptosis.

Kinase-inhibiting RNase-

attenuators (KIRAs): compounds that bind to the ATP-binding site and allosterically impede the RNase activity.

Kinase type I inhibitors: the type I inhibitors bind to the so called 'active conformation' of the enzyme, and are associated with the DFG-in conformation of this loop.

Kinase type II inhibitors: class of chemicals that trap their target kinases in an inactive, so-called DFG-out state by occupying a hydrophobic pocket adjacent to the ATP binding site. PROTACS: chimeric bifunctional molecules able to bind simultaneously the targeted protein, and a degrader protein, such as the ubiquitin E3 ligase, leading ultimately to the degradation and removal of the target from the cell.

Proteasome: multisubunit assembly of proteases that selectively degrades proteins, including transcription factors that regulate the cell cycle.

Schiff base: a compound with the general structure R C=NR'. They can be considered a subclass of imines, being either secondary ketimines or secondary aldimines depending on their structure. The term is often synonymous with azomethine which refers specifically to secondary aldimines.

Trans-autophosphorylation: the phosphorylation by a protein of a residue on an identical protein. For example, phosphorylation by the other kinase within a homodimer.

Unfolded protein response (UPR): cellular stress response related to ER stress. The UPR is activated in response to an accumulation of unfolded or misfolded proteins in the lumen of the ER. 
(A)

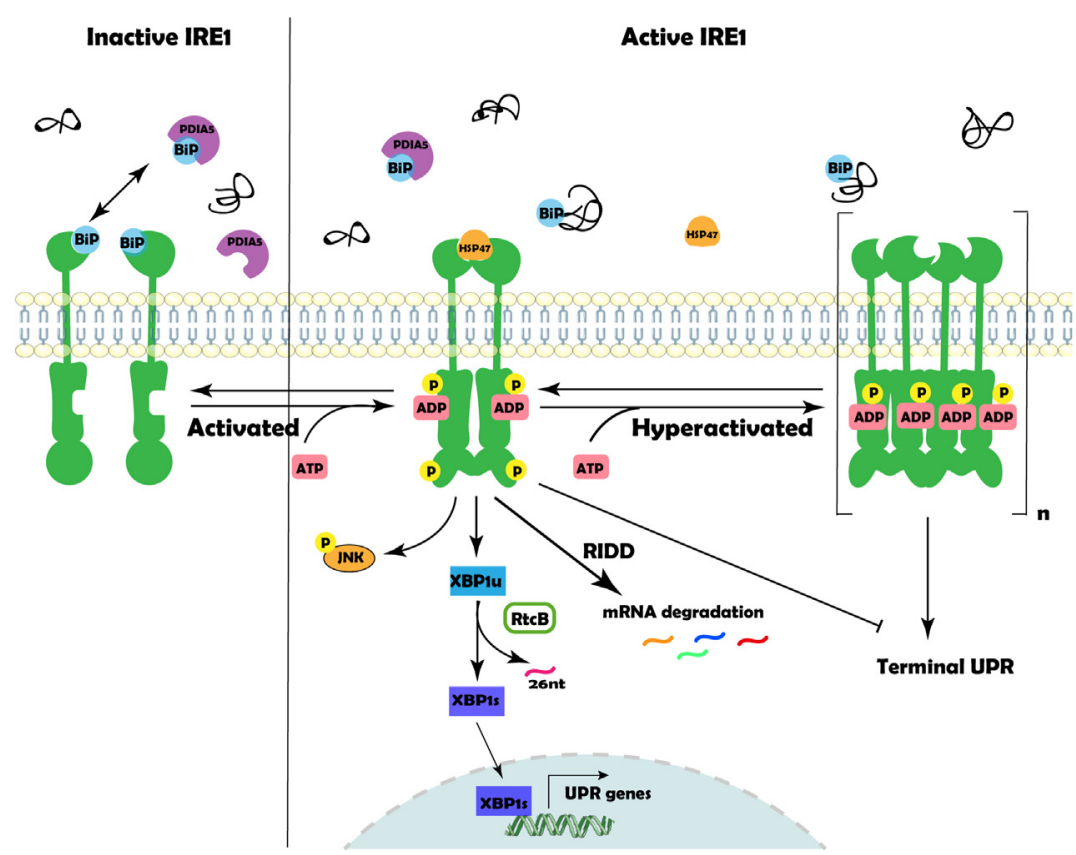

(B)
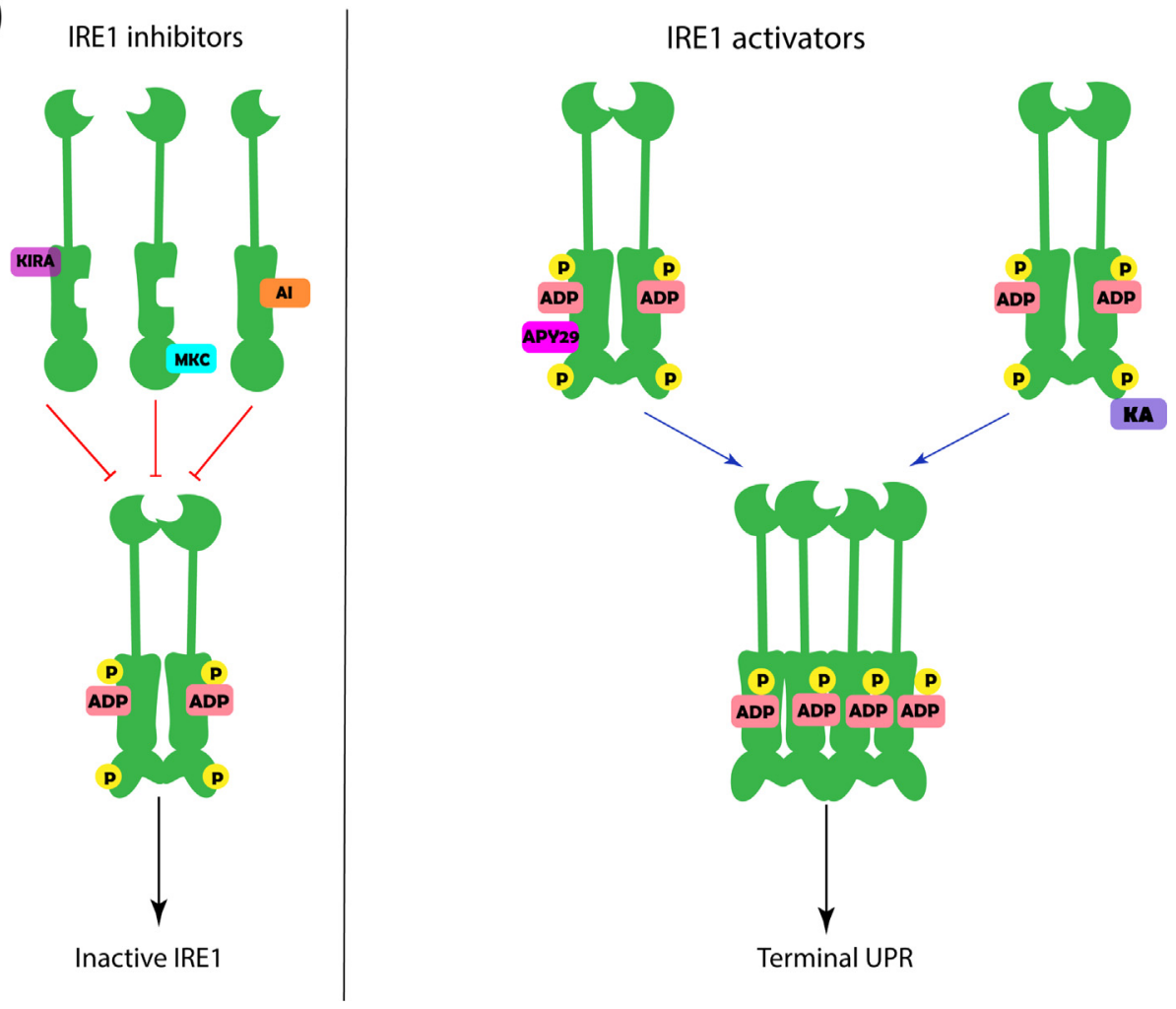

Trends in Cancer

(See figure legend at the bottom of the next page.) 


\begin{tabular}{|c|c|c|c|c|c|c|}
\hline Drug & Kinase activity & RNase activity & RIDD & Class & $\mathrm{EC}_{50}$ & Refs \\
\hline Cpd 3 & Inhibits & Inhibits & Unknown & Type II inhibitor & $3.12 \mu \mathrm{M}$ & [25] \\
\hline KIRA6 & Inhibits & Inhibits & Inhibits & Type II inhibitor & $0.17-0.87 \mu \mathrm{M}$ & [13] \\
\hline KIRA7 & Inhibits & Inhibits & Inhibits & Type II inhibitor & $0.11 / 0.22 \mu \mathrm{M}$ & {$[27]$} \\
\hline KIRA8 & Inhibits & Inhibits & Inhibits & Type II inhibitor & $0.007 \mu \mathrm{M}$ & [28] \\
\hline Cpd 31 & Inhibits & Inhibits & Unknown & $\begin{array}{c}\text { Imidazo[1,2-b] } \\
\text { pyridazin-8-amine }\end{array}$ & $0.008-0.78 \mu \mathrm{M}$ & [29] \\
\hline GSK2850163 & Inhibits & Inhibits & Unknown & $\begin{array}{l}\text { 2,7-Diazaspiro[4.5] } \\
\text { decane derivative }\end{array}$ & $0.02-0.2 \mu \mathrm{M}$ & [30] \\
\hline STF-083010 & No effect & Inhibits & Inhibits & $\begin{array}{l}\text { Inimine-based } \\
\text { compound }\end{array}$ & $25 \mu \mathrm{M}$ & {$[7]$} \\
\hline HNA (2-hydroxy-1-naphthaldehyde) & Unknown & Inhibits & Unknown & HAA & $31-35 \mu \mathrm{M}$ & {$[7]$} \\
\hline B-109 & Unknown & $\begin{array}{l}\text { Inhibition of } \\
\text { XBP1 splicing }\end{array}$ & Unknown & HAA & $1.23 \mu \mathrm{M}$ & [33] \\
\hline $4 \mu 8 \mathrm{C}$ & Inhibits & Inhibits & Inhibits & HAA & $0.076 \mu \mathrm{M}-6.9 \mu \mathrm{M}$ & {$[37]$} \\
\hline OICR573 & No effect & Inhibits & Unknown & HAA & $5.4 \pm 1.9 \mu \mathrm{M}$ & [32] \\
\hline OICR464 & No effect & Inhibits & Unknown & HAA & $2.3 \pm 0.69 \mu \mathrm{M}$ & [32] \\
\hline MKC-3946 & $\begin{array}{l}\text { No effect on } \\
\text { auto-phosphorylation }\end{array}$ & Inhibits & Unknown & HAA & $0.23 \pm 0.03 \mu \mathrm{M}$ & {$[7,34]$} \\
\hline MKC9989 & Unknown & Inhibits & Unknown & HAA & $0.29 \mu \mathrm{M}$ & [32] \\
\hline MKC8866 & Unknown & Inhibits & Inhibits & HAA & $0.39 \pm 0.21 \mu \mathrm{M}$ & [32] \\
\hline Toyocamycin & No effect & Inhibits & Unknown & Pyrrolopyrimidine & $0.08-0.18 \mu \mathrm{M}$ & {$[46]$} \\
\hline Doxorubicin & Unknown & Inhibits & No effect & Anthracycline antibiotic & $\sim 20 \mu \mathrm{M}$ & [53] \\
\hline 3-methoxy-6-bromosalicylaldehyde & Unknown & Inhibits & Unknown & Salicylaldehydes & $0.41 \mu \mathrm{M}$ & [39] \\
\hline Trierixin & Inhibits & Unknown & Unknown & Triene-ansamycin group & $14-19 \mathrm{ng} / \mathrm{mL}$ & [76] \\
\hline Quino-trierixin & Inhibits & Unknown & Unknown & Triene-ansamycin group & $0.067 \mu \mathrm{M}$ & [77] \\
\hline
\end{tabular}

[21-23]. Extensive crystallization efforts led to the obtention of crystal structures from yeast and human IRE1 in active and inactive forms, revealing its probable mechanism of activation through cofactor binding and trans-autophosphorylation, ultimately leading to the active back-to-back dimer [17]. These observations were supported by studies of mutant IRE1 proteins carrying amino acid substitutions at conserved positions in the kinase domain, which identified nucleotide binding and kinase-domain phosphorylation as necessary for IRE1 RNase activation [17,24].

\section{IRE1 Kinase Inhibitors}

Compound 3 is an optimized kinase type II inhibitor and one of the earliest reported ATPcompetitive inhibitors capable of decreasing RNase activity, and was part of a chemical series

Figure 1. IRE1 Signaling and Its Modulation. (A) The activation of IRE1 by autophosphorylation, dimerization, and oligomerization is regulated positively by many proteins (e.g., BiP and HSP47). Once activated, IRE1 triggers three main responses to ER stress: (i) apoptosis through the JNK pathway; (ii) activation of UPR genes through XBP1 splicing; and (iii) degradation of many mRNAs by RIDD. The stabilization of the oligomeric state leads to an inflammatory/mitochondrial programmed cell death via terminal UPR. (B) The inhibitors (left side) and activators (right side) of IRE1 can lead to sensitization of cells to chemotherapy by the IRE1 pathway. The inhibitors lead to an inactivation of IRE1 activity, inhibiting the response under ER stress. The overactivation of IRE1 leads to terminal UPR by activating nonreversible ER stress. Abbreviations: Al, ATP inhibitor; IRE1, inositol requiring enzyme 1; KA, kinase activator; JNK, c-Jun N-terminal kinase; RIDD, regulated IRE1-dependent decay; UPR, unfolded protein response; ER, endoplasmic reticulum. 


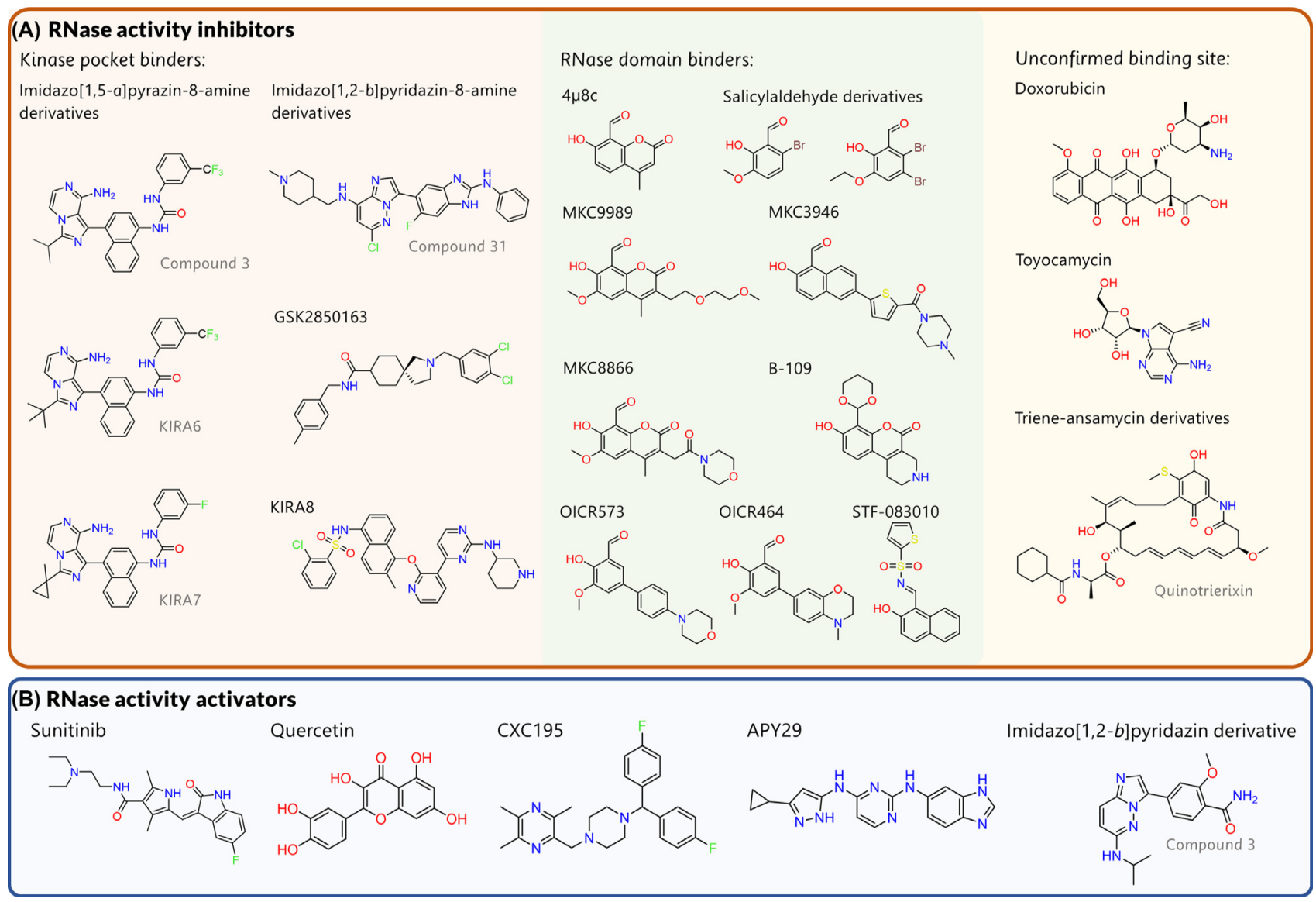

Trends in Cancer

Figure 2. Small Molecule Modulators of IRE1 a Activity. (A) Molecular structures of the most significant IRE1 inhibitors in their chemical series, classified by their binding sites: (i) kinase pocket binders; (ii) RNase domain ligands; and (iii) unconfirmed binding site inhibitors. (B) Molecular structures of IRE1 activators. Abbreviation: IRE1, inositol requiring enzyme 1; KIRA, kinase-inhibiting RNase-attenuator.

based on the imidazo[1,5-a]pyrazin-8-amine scaffold [25]. This type of compound presents the advantage of being often more specific than those targeting active, DFG-in, kinase conformations. The imidazo[1,5-a] pyrazine motif of Compound 3 forms two hydrogen bonds with the hinge region and occupies the adenine pocket of IRE1. This competition causes movements of the DFG-motif (adopting a DFG-out conformation) contained within the activation loop. As a consequence, Compound 3 inhibits XBP1 mRNA splicing, even during ER stress [25] (Table 1 and Figure 2A). Kinase-inhibiting RNase-attenuators (KIRAs) were recently developed based on Compound 3 and are highly efficient ligands mono-selective towards IRE1. The first KIRA was assayed using fluorescence resonance energy transfer (FRET)-quenched XBP1 RNA minisubstrate. The most potent compound identified in this study inhibited XBP1 RNA cleavage to a similar degree as STF-083010, a cell-permeable compound that directly targets the IRE1 RNase domain and disrupts IRE1-XBP1 activity [26]. KIRA compounds, as type II inhibitors, alter the oligomerization process on the DFG-motif in order to stabilize the enzyme in a helixaC-out conformation, which affects both kinase and RNase activities depending on the dose used. Optimized KIRA compounds include KIRA6 [13], KIRA7 [27], and KIRA8 [28]. KIRA6 and KIRA7 were developed on the basis of Compound 3 and the three molecules share high features similarity [13], whereas KIRA8 is a sulfonamide compound displaying high selectivity [28]. They were proven to protect pancreatic $\beta$-cells against autoimmune diabetes, halt neurodegeneration 
(A)

(B) KIRA8

(C) MKC 9989

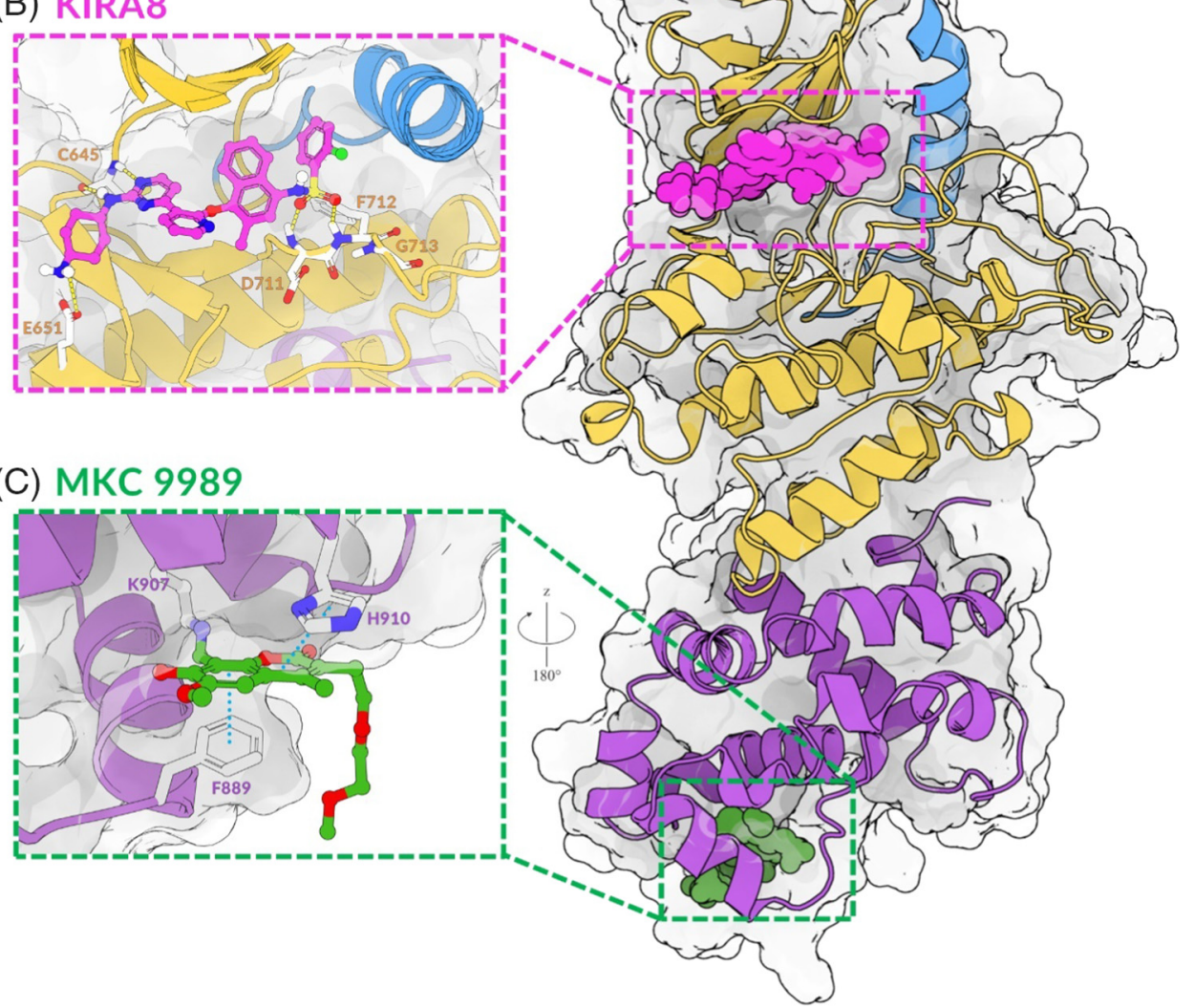

Trends in Cancer

Figure 3. IRE1 Targeting by Small-Molecule Inhibitors. (A) Graphical representation of kinase pocket inhibitors (pink) and RNase domain covalent inhibitors (green) bound to the IRE1 monomeric structure. (Composite structure generated for illustrative purpose using PDB ID 4U6R, in which MKC 9989 was docked using Schrodinger Maestro's CovDock; kinase domain represented in yellow, RNase domain in purple, and aC-helix in blue.) (B) X-ray crystal structure (PDB ID 4 U6R) of KIRA8 bound to IRE1 kinase pocket. Key residues of the protein are shown as white stick, and hydrogen interactions as yellow broken lines. (C) X-ray crystal structure (PDB ID 4PL3) of MKC 9989 covalently bound to Lys907 of IRE1 RNase domain. Key residues of the protein are shown as white stick, and pi interactions as cyan broken lines. Abbreviation: IRE1, inositol requiring enzyme 1; KIRA, kinase-inhibiting RNase-attenuator.

in amyotrophic lateral sclerosis (ALS) (KIRAs 6-8) [13,23], preserve the photoreceptor degeneration induced by ER stress (KIRA6), and have antifibrotic effects in the lung (KIRA 7) [27]. Evident side effects after systemic administration have yet to be reported. Following the discovery of the imidazo[1,2-b]pyridazin based RNase activators, extensive targeted development and structure activity relationships (SAR) studies led to a series of potent inhibitors which lock the kinase DFG motif in an unusual and inactive 'DFG-up' state [29]. Compound 31 and its analogues inhibited both tunicamycin- and thapsigargin-induced IRE1-dependent splicing of XBP1 luciferase fusion mRNA in HEK293 cells. In parallel, inhibition of tunicamycin-induced production of endogenous XBP1s mRNA was demonstrated in myeloma cells using reverse transcription (RT)qPCR at similar concentrations [29]. GSK2850163 was identified in a high throughput screen to discover new IRE1 selective inhibitors. It showed both RNase and kinase inhibition in a dose-dependent manner in myeloma cell lines [30]. 
IRE1 RNase Inhibitors

Additional modulators with a different mode of action were identified in high-throughput screens (HTS) using luciferase-based reporters in cell-based assays or recombinant proteins in vitro [31]. These so-called direct inhibitors share a common hydroxy-aryl-aldehyde (HAA) moiety which reacts selectively with a specific lysine (Lys907) in the RNase domain, thus forming a stable imine via Schiff base formation and effectively preventing ER stress-induced site-specific mRNA splicing. These include 4 $48 \mathrm{C}$, salicylaldehydes, HNA [7], OICR573 and OICR464 [32], B-I09 [33], STF083010 [7], and the MKC analogues: MKC9989 [32], MKC8866 [32], and MKC3946 [7,34] (Table 1 and Figure 2A). In the case of B-109 and STF-083010, a naturally occurring hydrolysis step takes place under physiological conditions to form, in situ, the reactive HAA motif. STF083010 was identified as an inhibitor of XBP1 mRNA splicing, activated by thapsigargin, tunicamycin, glucose deprivation, or severe hypoxia, and was reported to block IRE1 endonuclease activity without affecting its kinase activity. STF-083010 is now in preclinical trials for multiple myeloma (MM) and breast cancer treatment, displaying selective cytotoxicity towards cancer cells [26]. B-I09 inhibits growth of human chronic lymphocytic leukemia (CLL) cells in vitro, and promotes regression in a murine CLL model [33]. $4 \mu 8 \mathrm{C}$ also inhibits RIDD mRNA degradation in mouse embryonic fibroblast cultures following tunicamycin treatment [35]. Compared with STF-083010, 4 $\mu 8 \mathrm{C}$ was also shown to inhibit IRE1 autophosphorylation via Schiff base formation with IRE1 Lys599 located in the kinase pocket [36,37]. HNA (2-hydroxy-1-naphthaldehyde) is a small HAA molecule that showed either the same or more potent ability to inhibit the activity of IRE1, than the ability of MKC-3946 to cleave XBP1, after tunicamycin induced activation of acute myeloid leukemia cells (AML). The combination of bortezomib (BTZ), a proteasome inhibitor, or $\mathrm{AS}_{2} \mathrm{O}_{3}$ with $\mathrm{HNA}$, synergistically both inhibited growth of AML cells when applied to an AML patient sample $[7,38]$. $4 \mu 8 \mathrm{C}$ was tested in both cell-free assays and in cultured cells for potential activity as a reactive oxygen species (ROS) scavenger. It was demonstrated that this molecule can effectively decrease xanthine/xanthine oxidase catalyzed superoxide production in vitro, as well as inhibit angiotensin II-induced ROS production in vivo. In both cases, the concentration used is much lower than when used as a direct inhibitor of IRE1 [35]. MKC3946 is active in $\mathrm{MM}$ and reinforces the action of BTZ. Recent structural studies confirmed the interaction site between IRE1 Lys907 and HAA-based compounds MKC9989, MKC8866, OICR573, and OICR464. All four molecules were potent inhibitors of RNase activity in vitro. Salicylaldehydes were found to be selective and potent inhibitors of IRE1 endoribonuclease both in vitro and in vivo, competing against the XBP1 stem-loop RNA substrate [39]. 3-methoxy-6bromosalicylaldehyde could bind reversibly to IRE1 and block XBP1 mRNA splicing in different organs (kidney, liver, and spleen) after administration of tunicamycin [39]. It is likely that salicylaldehydes present the same binding mode with Lys907 as the other HAA-based inhibitors, but structural studies have yet to confirm this [40]. In addition to those direct modulators, other inhibitors with unconfirmed binding sites were identified. These molecules such as toyocamycin, doxorubicin, quinotrierixin, and trierixin, were shown to inhibit IRE1/XBP1s activity in vitro and in vivo, despite no clear demonstration of their mode of action.

\section{IRE1 RNase Activators}

The concept developed previously, is that inhibiting IRE1 activity might represent an interesting approach to weaken the adaptability of tumor cells, and therefore make them more sensitive to intrinsic modifications in their challenging microenvironment, or to the treatments they are exposed to. Another strategy could consist of initiating overwhelming stress signals that cause cell death. As such APY29 or sunitinib (both kinase type I inhibitors) are allosteric modulators inhibiting IRE1 trans-autophosphorylation, whilst promoting oligomerization and activation of its RNase activity $[23,41]$. Sunitinib inhibits tumor migration through remodeling of extracellular matrix and intracellular signaling pathways, such as metalloproteinases and kinases [25,37]. APY29 
decreased IRE1 ubiquitylation, and showed potent effects against sepsis in preclinical models of inflammation. Moreover, its interaction with Cys645 at the kinase cleft has been reported to activate IRE1 endoribonuclease activity [42]. CXC195 was first described to trigger the activation of many proteins related to ER stress, including BiP, CHOP, IRE1, TRAF2, p-ASK1, and p-JNK in bladder urothelial carcinoma cells. This induced apoptosis through stabilization of the IRE1TRAF2-ASK1 complex [11]. CXC195 was associated with proapoptotic effects in hepatocellular carcinoma cells due to activation of IRE1 and ATF6, and inhibition of the PI3K/Akt/mTOR signaling pathway [43]. Despite there being no direct evidence of the effect of this drug on IRE1 in this model, the findings show decreasing levels of Bax, an important activator of IRE1 activity. The mechanisms by which this molecule works remain to be elucidated. Prior to the development of the imidazo[1,2-b]pyridazin based RNase inhibitors [29](vide supra), a limited series of ligands based on this scaffold were identified as human isoform IRE1 RNase activators [24]. Among those, Compound 3 was found to enhance the endoribonuclease activity of unphosphorylated human IRE1 in vitro, with potency $\left(\mathrm{EC}_{50} 143 \mathrm{nM}\right)$ similar to the inhibition of the kinase autophosphorylation activity $\left(\mathrm{IC}_{50} 218 \mathrm{nM}\right)$ [24]. In this context, the identification of compounds that would therefore selectively activate a specific arm of the UPR (and the IRE1 pathway in particular) is of great importance. Finally, one might propose that an IRE1 activator may also boost the folding capacity of the ER, through enhanced adaptive signaling, which in turn could impact the rescuing loss-of-folding/secretory functions in protein misfolding/trafficking diseases (Table 2 and Figure 2B). The latter condition was actually recently illustrated by the identification of IRE1 activators independently of the UPR that can selectively reprogram ER proteostasis [79].

\section{Potential Off-Target Activities of IRE1-Targeting Compounds}

The off-target activity of a compound is defined as the activity that the compound could exert on an effector different from its intended biological target. Off-target effects commonly contribute to side effects. However, in some cases, off-target activity can be used as an advantage for therapeutic purposes. As such, the concept of polypharmacology has emerged to repurpose molecules that can exert activities on multiple targets, thus mediating beneficial effects [44]. In this context, drug repurposing represents an exciting opportunity to highlight the potential of approved drugs and small molecules on different targets. In this context, some of the compounds described in this review were not originally identified as IRE1-targeting compounds, but experiments revealed them to be effective IRE1 modulators. For instance, toyocamycin was first isolated as an antibiotic in 1956 and extracted from Streptomyces toyocaensis [45]. Since it is a nucleoside-type antibiotic analogue of adenosine, it was suggested that it could act as an ATPcompetitive inhibitor $[7,46]$. Sunitinib malate (Pfizer Sutent $\AA$; ClinicalTrials.gov number NCT00428597) was first identified as an oral multikinase inhibitor that works by blocking multiple molecular targets implicated in the growth, proliferation, and spread of cancers [47]. Two important sunitinib targets, vascular endothelial growth factor receptor (VEGFR) and platelet-derived growth factor receptor (PDGFR) are expressed by many types of solid tumors, and are thought to play a crucial role in angiogenesis. Sunitinib also inhibits other targets important to tumor growth, including KIT, FLT3, and RET [23]. Similar to sunitinib, APY-29 was identified at first as

Table 2. IRE1 Activators

\begin{tabular}{|c|c|c|c|c|c|c|c|}
\hline Drug & Kinase activity & RNase activity & RIDD & Class & EC \\
\hline APY29 & Activates & Unknown & Unknown & Type I kinase inhibitor & $0.28 \mu \mathrm{M}$ & {$[41]$} \\
\hline Sunitinib & Inhibits & Activates & Unknown & Tyrosine kinase (RTK) inhibitor & $3.7 \mu \mathrm{M} \pm 1.2$ & {$[23]$} \\
\hline CXC195 & Unknown & Stabilization of IRE1-TRAF2-ASK1 complex & Unknown & Tetramethylpyrazine analog & Unknown & {$[43]$} \\
\hline Compound 3 & Inhibits & Activates & Unknown & Imidazopyridazin & $0.143-0.218 \mu M$ & {$[24]$} \\
\hline
\end{tabular}


a broad-acting kinase inhibitor. However, its ability to activate UPR identified it as an allosteric modulator of IRE1 [48].

\section{Adjuvant Use of IRE1 Inhibitors in Cancer}

In tumors, upregulation of UPR markers is frequently observed, which could be caused by cancer specific stresses such as genomic instability, somatic mutations, oncogene expression, aneuploidy, or nutrient shortage. The UPR also contributes to cancer independently of protein misfolding, and ER stress is related to support, survival, and adaptation of cancer cells. IRE1 is emerging as a driver of migration, homing, and invasion of cancer cells. Its activity modulates the adhesion of glioblastoma multiform (GBM) cells [15], enhances metastasis of triple-negative breast cancer (TNBC) tumors to the lungs [14], and is related to chemoresistance in many types of cancers such as breast cancer, prostate cancer, MM, leukemia, and glioblastoma $[3,7,10,14,34,46,49,50]$. In this context, there have been many attempts to improve existing clinical treatments by coadministering IRE1 inhibitors (Table 3). Monotherapies with IRE1 inhibitors, or in combination with antitumorigenic agents, significantly reduced tumor growth of mice bearing human prostate tumors [51]. In TNBC, the breast cancer type that typically shows worst prognosis, IRE1 RNase activity enhances the production of the protumorigenic factors interleukin (IL)-6, IL-8, CXCL1, granulocyte-macrophage colony-stimulating factor (GM-CSF), and transforming growth factor (TGF) $\beta 2$, and its inhibition can sensitize TNBC cells to the chemotherapeutic, paclitaxel [14]. MM is an extremely aggressive, incurable, and mostly lethal disease despite advances in therapy. It arises from malignant immunoglobulin (lg)-secreting plasma cells. Pharmacological inhibition of the IRE1-XBP1s pathway has been demonstrated as an effective and safe potential small molecule target for MM therapy [52]. Toyocamycin $[7,46]$ was described as a cytotoxic agent for MM cells, and was shown to selectively affect IRE1 signaling but not PERK or ATF6 signaling. The adenine moiety of toyocamycin seems to inhibit XBP1 activation, and the combination of this drug with BTZ markedly induced apoptosis in BTZ-resistant MM cells. Furthermore, anthracyclines such as doxorubicin have proven useful in MM and in combinations with BTZ. Doxorubicin is a chemotherapy medication used to treat many types of cancer. It acts by intercalating DNA/RNA and inhibiting topoisomerase II. Similarly to toyocamycin, it displayed increased cytotoxicity against myeloma compared with other cancer cell lines which have lower basal IRE1XBP1 activity [53]. Finally, GBM is an aggressive and fatal central nervous system tumor, in which

Table 3. Combined Treatments with IRE1 Inhibitors

\begin{tabular}{|c|c|c|}
\hline Treatments & Cancer & Refs \\
\hline Compound $18+$ anti-VEGF-A & TNBC & {$[49]$} \\
\hline MKC-8866 + Paclitaxel & TNBC & {$[14]$} \\
\hline MKC-3946 + Bortezomib & MM & {$[34]$} \\
\hline MKC-3946 + 17AAG & MM & {$[34]$} \\
\hline STF-083010 + Tamoxifen & Breast Cancer & {$[50]$} \\
\hline MKC-8866 + Enzalutamide & PCa & {$[3]$} \\
\hline MKC-8866 + Abiraterone acetate & PCa & {$[3]$} \\
\hline MKC-8866 + Cabazitaxel & PCa & {$[3]$} \\
\hline MKC-8866 + Docetaxel & Breast Cancer & {$[10]$} \\
\hline Toyocamycin + BTZ & MM & {$[46]$} \\
\hline Doxorubicin + BTZ & MM & {$[78]$} \\
\hline HNA + Bortezomib & AML & {$[7]$} \\
\hline HNA + AS $\mathrm{O}_{3}$ & AML & {$[7]$} \\
\hline MKC8866 + Stupp & GBM & {$[54]$} \\
\hline
\end{tabular}


downstream IRE1 signaling commands tumor angiogenesis, invasion, and migration [15]; recently the adjuvant use of MKC8866 was found to enhance the efficacy of irradiation combined with temozolomide treatment [54].

\section{IRE1 Signaling Cross-Talks with Other Stress Pathways}

IRE1 signaling has been shown to crosstalk with many cellular stress pathways. Consequently, one can expect that the modulation of IRE1 signaling could also influence other cancer-relevant pathways in malignant cells, and IRE1-targeting compounds could therefore gain importance in the impairment of tumorigenesis. Proposed herein is a description of the cross-talks between IRE1 signaling and pathways involved in the hallmarks of cancer [55], and a discussion on how modulating IRE1 could regulate them.

Initiation is the first step in the two-stage model of cancer development. Initiators cause irreversible changes (mutations) to DNA, thereby increasing cancer risk. To avoid an enormous amount of DNA mutations, surveillance proteins monitor DNA integrity, and activate cell cycle checkpoints and DNA repair pathways in response to DNA damage. IRE1 seems to modulate the DNA damage response through RIDD. Promotion is the second stage of cancer development in which IRE1 signaling is known to play instrumental roles [56]. Consequently, the IRE1/XBP1 axis is: (i) activated in response to nutrient starvation, and controls the expression of limiting enzyme of the hexosamine biosynthetic pathway (HBP) activation. This allows the metabolism of glucose to UDP-acetylglucosamine, which serves as a substrate for $\mathrm{O}$ - and $\mathrm{N}$-linked glycosylation which promotes proteostasis [57]; (ii) cross-talks with the hypoxia-inducible factor alpha (HIF1a) pathway, which in turn induces glycolysis and response to hypoxia [58,59]; (iii) controls the transcription of genes coding for VEGF-A, IL-6, and other proangiogenic signals leading to tumor neo-angiogenesis [14]; and (iv) controls the induction of tumor adaptive autophagy through the expression of Beclin-1 [60]. Alternatively, IRE1 can also exert other procancer-related functions through RIDD or scaffolding activities. Indeed, RIDD activity controls the expression of SPARC (secreted protein acidic and rich in cysteine) mRNA in glioblastoma, thus altering migration and invasion properties via the activation of the small GTPase RhoA [61,62]. Moreover, IRE1 directly binds filamin $A$ to regulate the remodeling of the cytoskeleton and impact on cell migration [63].

The effect of IRE1 modulation is dependent on the type of cancer, and studies on the precise roles of IRE1 in various cancers are essential for relevant therapeutic intervention. For instance, studies have shown that high levels of the major components of UPR (PERK, ATF6, IRE1) and both unspliced and spliced XBP1, are observed in a variety of human tumors including brain, breast, gastric, kidney, liver, lung, and pancreatic cancer [13-15,27,36,49,57,58]. In addition, activation of IRE1 signaling led to a reduction in colorectal tumor growth and increased survival in mouse models [59], indicating that knowledge must drive the use of IRE1 targeting drugs. Hence, it appears that targeting IRE1 can have anticancer effect at both the level of the tumor cells themselves and the antitumor capacity of other stress pathways.

\section{Concluding Remarks}

The ER controls multiple cellular functions including protein folding, post-translational modifications (PTMs), fatty acid biosynthesis, detoxification, and intracellular calcium storage. As such it plays an instrumental role in tumor development and treatment response. A large panel of modulators of IRE1 activity, through the targeting of its catalytic activities, have been described earlier. In this context, one should be cautious with the use of those pharmacological modulators, as they might exert deleterious effects on normal tissues, in particular those involved in secretory functions (e.g., liver, pancreas, and B cells). For instance, inhibition of PERK, another UPR sensor,
Outstanding Questions How does IRE1 act on tumor progression in different types of cancer?

Can we use protein structure, rational drug design, and artificial intelligence to predict new molecules that modify the responses of the pathways? 
by GSK2656157 in various in vivo cancer models [64], has shown some effects on tumor growth, however, long term administration of this compound also exerted significant side effects, including weight loss and mild diabetes, caused by damage to the pancreas, which challenged its use in the clinic. An IRE1 RNase inhibitor (MKC8866) has been extensively used in mouse orthotopic or xenograft models, in immunocompetent or deficient backgrounds, and thus far deleterious side effects have not been observed [14,54]. It is possible that under specific physiological conditions (e.g., infection and metabolic challenge), those inhibitors might lead to altered immune or inflammatory responses, but this should be examined thoroughly.

IRE1 functions can also be exerted through the scaffolding of partner proteins and as such, modulating the IRE1 interactome might represent another way to reprogram IRE1 functions. The many protein-protein interactions (PPIs) involving IRE1, with itself during the oligomerization process, or with partners such as BiP, HSP47, and the Sigma1 receptor, which fine-tune its activation level, Filamin A, or IP3 Receptors, may represent appealing opportunities to develop PPI inhibitors or stabilizers $[60,65,66]$. PROTACS (proteolysis targeting chimeras) and other chimeric degrader solutions [67] figure among the most trending novel therapeutic modalities as they present several advantages over small-molecule inhibition and allow for fine control over the cellular level of targeted protein, and have already been applied with success to transmembrane proteins with enzymatic activities similar to IRE1 [68]. The wide range of high-affinity IRE1 ligands available, makes PROTACS a compelling option to pursue. To overcome the limitations of previously described kinase and RNase binding modulators, finding molecular starting points that bind to other sites of the protein would be extremely valuable in the development of novel allosteric inhibitors or PPI modulators. Fragment-based drug discovery approaches have proven to be particularly successful in identifying small chemical entities binding to such novel binding sites, and represent another avenue worth exploring $[69,70]$.

Until now, efforts to improve cancer treatments by exploiting IRE1 activity have been restricted to its inhibition. Indeed, the activation of IRE1 signaling under low ER stress promotes autophagy and cell survival through an XBP1s mechanism. However, it has been described that under irrevocable ER stress, IRE1 oligomerizes to activate RIDD, ultimately leading to apoptosis [13]. Other death receptors such as RIP1 show the same switching mechanism process, which depends on the context or strength of cellular processes $[71,72]$. Induced hyper activation of IRE1 to convert the response from promoting homeostasis to promoting cell death, could thus represent an alternative strategy in cancer treatments [13]. Furthermore, IRE1 mutants in cancer cells show defective homo-oligomerization and RNase activity, which may allow the terminal UPR to become disabled or co-opted for survival advantage [73]. Based on this knowledge and the trends observed in the field recently, the discovery of novel families of IRE1 modulators as well as drug repurposing and combined therapies appear imminent as well as pertinent. In addition to these approaches, the rapid rise and improvements demonstrated by artificial intelligence methods hold great promise and could accelerate significantly the drug discovery process, from the design stage to the prediction of the most cost- and time-efficient synthesis pathways $[74,75]$ (see Outstanding Questions).

\section{Acknowledgments}

This work was funded by grants from Institut National du Cancer (INCa PLBIO), Fondation pour la Recherche Médicale (FRM, équipe labellisée 2018), ERANET, and Agence Nationale de la Recherche (ERAAT) to E.C.; EU H2020 MSCA ITN675448 (TRAINERS), and MSCA RISE-734749 (INSPIRED) provided grants to L.A.E. and E.C. D.P.R. was funded by INSERM (LA VANNETAISE). X.G. was funded by a post-doctoral fellowship from Fondation ARC, and D.D. and A.C. were MSCA ITN fellows (TRAINERS). 


\section{References}

1. Urra, H. et al. (2016) Endoplasmic reticulum stress and the hallmarks of cancer. Trends Cancer 2, 252-262

2. Urra, H. et al. (2013) When ER stress reaches a dead end. Biochim. Biophys. Acta 1833, 3507-3517

3. Sheng, X. et al. (2019) IRE1 $\alpha-X B P 1$ s pathway promotes prostate cancer by activating c-MYC signaling. Nat. Commun. 10, 323

4. Beaupere, C. et al. (2018) Genetic screen identifies adaptive aneuploidy as a key mediator of ER stress resistance in yeast. Proc. Natl. Acad. Sci. U. S. A. 115, 9586-9591

5. Terhorst, A. et al. (2020) The environmental stress response causes ribosome loss in aneuploid yeast cells. Proc. Natl. Acad. Sci. U. S. A. 117, 17031-17040

6. Amawi, H. et al. (2018) Bax/tubulin/epithelial-mesenchyma

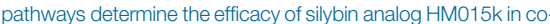
lorectal cancer cell growth and metastasis. Front. Pharmacol. 9 , 520

7. Sun, $\mathrm{H}$. et al. (2016) Inhibition of IRE1a-driven pro-survival pathways is a promising therapeutic application in acute myeloid leukemia [published correction appears in Oncotarget. 2017 Sep 8;8(38):64651]. Oncotarget 7, 18736-18749

8. Shi, W. et al. (2019) Unravel the molecular mechanism of XBP1 in regulating the biology of cancer cells. J. Cancer 10 , 2035-2046

9. Leary, M. et al. (2018) Sensitization of drug resistant cancer cells: A matter of combination therapy. Cancers (Basel) 10, 483

10. Zhao, N. et al. (2018) Pharmacological targeting of MYC regulated IRE1/XBP1 pathway suppresses MYC-driven breast cancer. J. Clin. Invest. 128, 1283-1299

11. Zeng, T. et al. (2015) IRE1 a-TRAF2-ASK1 complex-mediated endoplasmic reticulum stress and mitochondrial dysfunction contribute to CXC195-induced apoptosis in human bladde carcinoma T24 cells. Biochem. Biophys. Res. Commun. 460, 530-536

12. Carreras-Sureda, A et al. (2019) Non-canonical function of IRE1 a determines mitochondria-associated endoplasmic reticulum composition to control calcium transfer and bioenergetics. Nat. Cell Biol. 21, 755-767

13. Ghosh, R. et al. (2014) Allosteric inhibition of the IRE1a RNase preserves cell viability and function during endoplasmic reticulum stress. Cell 158, 534-548

14. Logue, S.E. et al. (2018) Inhibition of IRE1 RNase activity modulates the tumor cell secretome and enhances response to chemotherapy. Nat. Commun. 9

15. Lhomond, S. et al. (2018) Dual IRE 1 RNase functions dictate glioblastoma development. EMBO Mol. Med. 10, 3267

16. Storm, M. et al. (2016) Prostate cancer and the unfolded protein response. Oncotarget 7, 54051-54066

17. Korennykh, A. and Walter, P. (2012) Structural basis of the unfolded protein response. Annu. Rev. Cell Dev. Biol. 28, 251-277

18. Lee, K.P.K. et al. (2008) Structure of the dual enzyme Ire1 reveals the basis for catalysis and regulation in nonconventional RNA splicing. Cell 132, 89-100

19. Dhanasekaran, D.N. and Reddy, E.P. (2008) JNK signaling in apoptosis. Oncogene 27, 6245-6251

20. Han, Y. et al. (2014) Structure of human RNase $L$ reveals the basis for regulated RNA decay in the IFN response. Science $343,1244-1248$

21. Carlesso, A. et al. (2018) Binding analysis of the Inositol-requiring enzyme 1 kinase domain. ACS Omega 3, 13313-13322

22. Harrington, P.E. et al. (2015) Unfolded protein response in cancer: IRE1 a inhibition by selective kinase ligands does not impair tumor cell viability. ACS Med. Chem. Lett. 6, 68-72

23. Feldman, H.C. et al. (2016) Structural and functional analysis of the allosteric inhibition of IRE1 a with ATP-competitive ligands. ACS Chem. Biol. 11, 2195-2205

24. Joshi, A. et al. (2015) Molecular mechanisms of human IRE1 activation through dimerization and ligand binding. Oncotarget 6 , 13019-13035

25. Wang, L. et al. (2012) Divergent allosteric control of the IRE1a endoribonuclease using kinase inhibitors. Nat. Chem. Biol. 8 982-989

26. Papandreou, I. et al. (2010) Identification of an Ire1 alpha endonuclease specific inhibitor with cytotoxic activity against human multiple myeloma. Blood 117, 1311-1314
27. Thamsen, M et al. (2019) Small molecule inhibition of IRE1 a kinase/ RNase has anti-fibrotic effects in the lung. PLoS One 14 e0209824

28. Morita, S. et al. (2017) Targeting ABL-IRE1a signaling spares ER-stressed pancreatic $\beta$ cells to reverse autoimmune diabetes. Cell Metab. 25, 883-897

29. Colombano, G. et al. (2019) Binding to an unusual inactive kinase conformation by highly selective inhibitors of Inositolrequiring enzyme 1 a kinase-endoribonuclease. J. Med. Chem. 62, 2447-2465

30. Concha, N.O. et al. (2015) Long-range inhibitor-induced conformational regulation of human IRE1 a endoribonuclease activity. Mol. Pharmacol. 88, 1011-1023

31. Doultsinos, D. et al. (2017) Control of the Unfolded protein re sponse in health and disease. SLAS Discov. 22, 787-800

32. Sanches, M. et al. (2014) Structure and mechanism of action of the hydroxy-aryl-aldehyde class of IRE1 endoribonuclease inhibitors. Nat. Commun. 5, 1-16

33. Tang, C.A. et al. (2014) Inhibition of ER stress-associated IRE-1 XBP-1 pathway reduces leukemic cell survival. J. Clin. Invest. $124,2585-2598$

34. Mimura, N. et al. (2012) Blockade of XBP1 splicing by inhibition of IRE1 $\alpha$ is a promising therapeutic option in multiple myeloma. Blood 119, 5772-5781

35. Chan, S.M.H. et al. (2018) The Inositol-requiring enzyme (IRE1a) RNAse inhibitor, 4m8C, is also a potent cellular antioxidant. Biochem. J. 475, 923-929

36. Stewart, C. et al. (2017) Regulation of IRE1a by the small molecule inhibitor $4 \mu 8 \mathrm{c}$ in hepatoma cells. Endoplasmic Reticulum Stress Dis. 4, 1-10

37. Cross, B.C.S. et al. (2012) The molecular basis for selective inhibition of unconventional mRNA splicing by an IRE1-binding smal molecule. Proc. Natl. Acad. Sci. 109, E869-E878

38. Logue, S.E. et al. (2013) New directions in ER stress-induced cell death. Apoptosis 18, 537-546

39. Volkmann, K. et al. (2011) Potent and selective inhibitors of the Inositol-requiring enzyme 1 endoribonuclease. J. Biol. Chem. 286, 12743-12755

40. Carlesso, A. and Eriksson, L.A. (2019) Selective inhibition of IRE1 signalling mediated by MKC9989: new insights from molecular docking and molecular dynamics simulations. ChemistrySelect 4, 3199-3203

41. Korennykh, A.V. et al. (2009) The unfolded protein response signals through high-order assembly of Ire1. Nature 457 , 687-693

42. Rosen, D.A. et al. (2019) Modulation of the sigma-1 receptorIRE1 pathway is beneficial in preclinical models of inflammation and sepsis. 11, eaau5266

43. Chen, X.L. et al. (2015) CXC195 induces apoptosis and endoplastic reticulum stress in human hepatocellular carcinoma cells by inhibiting the PI3K/Akt/mTOR signaling pathway. Mol. Med. Rep. 12, 8229-8236

44. Ashburn, T.T. and Thor, K.B. (2004) Drug repositioning: identifying and developing new uses for existing drugs. Nat. Rev. Drug Discov. 3, 673-683

45. Nishimurah, H. et al. (1956) Toyocamycin, a new anti-candida antibiotics. J. Antibiot. (Tokyo) 9, 60-62

46. Ri, M. et al. (2012) Identification of toyocamycin, an agent cytotoxic for multiple myeloma cells, as a potent inhibitor of ER stress-induced XBP1 mRNA splicing. Blood Cancer J. 2, e79

47. Raymond, E. et al. (2011) Sunitinib malate for the treatment of pancreatic neuroendocrine tumors. N. Engl. J. Med. 364 501-513

48. Zhu, X. et al. (2018) Quercetin ameliorates HFD-induced NAFLD by promoting hepatic VLDL assembly and lipophagy via the IRE1a/XBP1s pathway. Food Chem. Toxicol. 114, 52-60

49. Harnoss, J.M. et al. (2020) IRE1a disruption in triple-negative breast cancer cooperates with anti-angiogenic therapy by reversing ER stress adaptation and remodeling the tumor microenvironment. Cancer Res. Published online April 7, 2020. https://doi.org/10.1158/0008-5472.CAN-19-3108

50. Ming, J. et al. (2015) A novel chemical, STF-083010, reverses tamoxifen-related drug resistance in breast cancer by inhibiting RE1/XBP1. Oncotarget 6, 40692-40703 
51. Sheng, X. et al. (2015) Divergent androgen regulation of unfolded protein response pathways drives prostate cancer. EMBO Mol. Med. 7, 788-801

52. Harnoss, J.M. et al. (2019) Disruption of IRE1 a through its kinase domain attenuates multiple myeloma. Proc. Natl. Acad. Sci. U. S. A. 116, 16420-16429

53. Jiang, D. et al. (2016) Identification of doxorubicin as an inhibitor of the IRE1 $\alpha-X B P 1$ axis of the unfolded protein response. Sci. Rep. 6, 1-8

54. Le Reste, P.J. et al. (2019) Development of a novel preclinica glioblastoma mouse model and therapeutic impact of IRE inhibition. bioRxiv Published online November 14, 2019. https://doi.org/10.1101/841296

55. Hanahan, D. and Weinberg, R.A. (2011) Hallmarks of cancer: the next generation. Cell 144, 646-674

56. Madden, E. et al. (2019) The role of the unfolded protein response in cancer progression: from oncogenesis to chemoresistance. Biol. Cell. 111, 1-17

57. Castillo, K. et al. (2011) BAX inhibitor-1 regulates autophagy by controlling the IRE1alpha branch of the unfolded protein response. EMBO J. 30, 4465-4478

58. Hetz, C. et al. (2006) Proapoptotic BAX and BAK modulate the unfolded protein response by a direct interaction with IRE1a. Science 312, 572-576

59. Rubio-Patiño, C. et al. (2018) Low-protein diet induces IRE1adependent anticancer immunosurveillance. Cell Metab. 27, 828-842.e7

60. Arkin, M.R. et al. (2014) Small-molecule inhibitors of proteinprotein interactions: progressing toward the reality. Chem. Biol. $21,1102-1114$

61. Auf, G. et al. (2010) Inositol-requiring enzyme $1 \alpha$ is a key regula tor of angiogenesis and invasion in malignant glioma. Proc. Natl. Acad. Sci. U. S. A. 107, 15553-15558

62. Dejeans, N. et al. (2012) Autocrine control of glioma cells adhesion and migration through IRE1a-mediated cleavage of SPARC mRNA. J. Cell Sci. 125, 4278-4287

63. Urra, H. et al. (2018) IRE1a governs cytoskeleton remodelling and cell migration through a direct interaction with filamin A. Nat. Cell Biol. 20, 942-953

64. Atkins, C. et al. (2013) Characterization of a novel PERK kinase inhibitor with antitumor and antiangiogenic activity. Cancer Res. 73, 1993-2002
65. Korennykh, A.V. et al. (2011) Cofactor-mediated conformational control in the bifunctional kinase/RNase Ire1. BMC Biol. 9, 48

66. Scott, D.E. et al. (2016) Small molecules, big targets: drug discovery faces the protein-protein interaction challenge. Nat Rev. Drug Discov. 15, 533-550

67. Churcher, I. (2018) Protac-induced protein degradation in drug discovery: breaking the rules or just making new ones? J. Med. Chem. 61, 444-452

68. Bondeson, D.P. et al. (2015) Catalytic in vivo protein knockdown by small-molecule PROTACs. Nat. Chem. Biol. 11, 611-617

69. Erlanson, D.A. et al. (2016) Twenty years on: the impact of fragments on drug discovery. Nat. Rev. Drug Discov. 15 605-619

70. Erlanson, D.A. et al. (2020) Fragment-to-lead medicinal chemistry publications in 2018. J. Med. Chem. 63, 4430-4444

71. Festjens, N. et al. (2007) RIP1, a kinase on the crossroads of a cell's decision to live or die. Cell Death Differ. 14, 400-410

72. Ofengeim, D. and Yuan, J. (2013) Regulation of RIP1 kinase signalling at the crossroads of inflammation and cell death. Nat. Rev. Mol. Cell Biol. 14, 727-736

73. Greenman, C. et al. (2007) Patterns of somatic mutation in human cancer genomes. Nature 446, 153-158

74. Mak, K.K. and Pichika, M.R. (2019) Artificial intelligence in drug development: present status and future prospects. Drug Discov. Today 24, 773-780

75. Struble, T.J. et al. (2020) Current and future roles of artificial intelligence in medicinal chemistry synthesis. J. Med. Chem. Published online April 3, 2020. https://doi.org/10.1021/acs. jmedchem.9b02120

76. Tashiro, E. et al. (2007) Trierixin, a novel inhibitor of ER stressinduced XBP1 activation from Streptomyces sp. J. Antibiot. 60, 582-585

77. Yamamoto, K. et al. (2011) Quinotrierixin inhibited ER stressinduced XBPI mRNA splicing through inhibition of protein synthesis. Biosci. Biotechnol. Biochem. 75, 284-288

78. Palumbo, A et al. (2008) Bortezomib, doxorubicin and dexamethasone in advanced multiple myeloma. Ann. Oncol. 19, $1160-1165$

79. Grandjean J.M.D., et al. Pharmacologic IRE1/XBP1s activation confers targeted ER proteostasis reprogramming. Nat. Chem. Biol. Published online July 20, 2020. https://dx.doi.org/ 10.1038/s41589-020-0584-z 